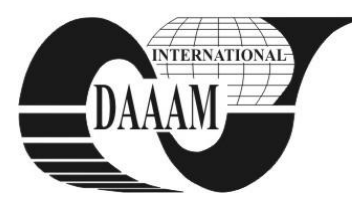

Annals of DAAAM for 2011 \& Proceedings of the 22nd International DAAAM Symposium, Volume 22, No. 1, ISSN 1726-9679 ISBN 978-3-901509-83-4, Editor B. Katalinic, Published by DAAAM International, Vienna, Austria, EU, 2011 Make Harmony between Technology and Nature, and Your Mind will Fly Free as a Bird Annals \& Proceedings of DAAAM International 2011

\title{
MANUFACTURING OPTIMIZATION WITH THE USE WORKSHOP MANUFACTURING PROCESS
}

\author{
WESSELY, E[mil]; BALCZAK, S[tanislav]; HRUBINA, K[amil] \& MACUROVA, A[nna]
}

\begin{abstract}
In the text the optimization of the manufacturing process is calculate by means the theory operatore analysis with assistence the copmuter. In the paper, it is proved that by means of the given algorithms of operational analysis methods it is possible to optimize a current manufacturing process by its replacement for a workshop manufacturing process. The calculations were performed numerically and based on the application of the MS Excel program.
\end{abstract}

Key words: optimization, operatore analysis, manufacturing process

\section{INTRODUCTION}

The problem is the control of the manufacturing process. Nowadays, most plants use a push system of a manufacturing control. It is controlled based on the schedule, which defines the start of individual activities. The coordination is provided merely based on capacity planning and a system control. Complexity and variability of individual processes lead to unexpected results which may be a consequence of an excessive stock of input materials, semi-finished products and complete products. In the plant running based on a push production control, i.e. if material flow and information flow are oriented in one direction, the task was to change the character of the control for the purpose of optimization in order to make the manufacturing process more effective in comparison with a current state. An important part of the scheduling system is optimization for which heuristic optimizing algorithms were designed. Thus, the result of optimization can be not only an optimized manufacturing layout, but an optimized configuration of a manufacturing system as well. This is especially important with flexible manufacturing systems which are designed so that they can be adapted to the changing manufacturing requirements (Katalinic, 1998).

\section{THEORETICAL BASIC}

In the next text the optimization of the manufacturing process is solution of the using operator analysis. If $c_{j}$ is the price of the $j$-th product and $x_{j}$ is its manufactured volume per time unit, the production value per time unit, i.e. cost function, is given by the relation (Balczak \& al 2010)

$$
J(x)=c_{1} x_{1}+c_{2} x_{2}+\ldots+c_{n} x_{n}
$$

Assumptions about the relations between a customer and a supplier result in a set of equations containing $m+n$ equations with $m . n$ variables. The task is to determine the value of the variables $x_{i j}$ that minimize costs of customer's orders handling

$$
J=\sum_{i=1}^{m} \sum_{j=1}^{n} q_{i j} x_{i j}
$$

The obtained results and calculations are given with the assistance the computer. The variable $q$ expressed by the following relation

$$
q=e \cdot\left(\frac{v}{a}\right)+y \cdot E \cdot T+d+z \cdot c+(b-1) \cdot c+r+u
$$

provides the basis for a mathematical expression of products distribution optimization. By means of the relation we calculate the time $q$ of the card path inside the regulation loop. Based on this, we calculate individual partial time sections $q_{\mathrm{i} \text { j }}$ that are used to calculate products distribution optimization by means of linear programming. The results of the card path inside the regulation loop are expressed in seconds (s), $q$ is time spent to process a file card (known in practice as time of a card circulation in a loop).

The variables meaning and some standard values of variables of the relation (1): $v$ time spent on manufacturing, $r$ possible repair, $z$ number of preparatory plants, $T=v / A$ time of all ordered products, manufacturing (in practice called takt time), $A$ customer's total demands, $a$ customer's demands, $b$ number of pieces /packages, $c$ cycle, $d$ version change, $w$ $10 \%$ time needed to change the version manufacturing time, $E$ batch dimension, $e=d /(w c)$ batch dimension, $e / b$ number of file cards batch dimension.

\begin{tabular}{|c|c|c|r|r|r|r|r|r|r|r|r|r|}
\hline$q_{i j}$ & e & v & a & \multicolumn{1}{c|}{$f$} & y & E & T & d & z & c & b-1 & r \\
\hline q11 & 50 & 27000 & 300 & 4500 & 3 & 50 & 90 & 200 & 3 & 40 & 3 & 200 \\
\hline q12 & 50 & 27000 & 100 & 13500 & 3 & 50 & 270 & 200 & 3 & 40 & 3 & 200 \\
\hline $\mathrm{q} 13$ & 50 & 27000 & 0 & 0 & 3 & 50 & 0 & 200 & 3 & 40 & 3 & 200 \\
\hline $\mathrm{q} 21$ & 50 & 27000 & 0 & 0 & 3 & 50 & 0 & 200 & 3 & 40 & 3 & 200 \\
\hline $\mathrm{q} 22$ & 50 & 27000 & 500 & 2700 & 3 & 50 & 54 & 200 & 3 & 40 & 3 & 200 \\
\hline $\mathrm{q} 23$ & 50 & 27000 & 100 & 13500 & 3 & 50 & 270 & 200 & 3 & 40 & 3 & 200 \\
\hline $\mathrm{q} 31$ & 50 & 27000 & 0 & 0 & 3 & 50 & 0 & 200 & 3 & 40 & 3 & 200 \\
\hline
\end{tabular}

Tab. 1. Partial time $q$ of the formule (1)

Kanban is preferably applied to workshops, where the material flow and individual manufacturing operations can be immediately adjusted to the imminent demand. For this reason, only demanded items are manufactured and shipped. The entire manufacturing control is subjected to a final assembly. Between the final assembly and previous work centres there are the so called automated regulation loops or independent manufacturing units. Regulation loops are created between a certain given resource and the manufacturing eventually assembly area and a central warehouse. The system was primarily determined for a control within one work area, but it can also be used for the control between individual work areas of the plant and even outside the plant. The most suitable application of the system is for a repetitive manufacturing of identical parts with a continuous and fairly steady demand. In other case, the system has to be provided with a special scheduling system. The system can be introduced in the plant under the following conditions

- High repetitiveness of manufacturing 
- Well trained and motivated staff

- Staff flexible responses to the changes in the process

- Levelling of the material consumption in the manufacturing process

- Quality control directly in the workplace

\section{DEGENERATE PROBLEM}

In practice, in comparison with the above described case, we encounter a degenerate problem. When analyzing the given problem, we evaluate if the problem was not degenerate which can occur if in some basic solution there are less than $(m+n-1)$ non-zero variables $x_{i j}$. The two calculations have been carried out in this work. The first one was related to the case, where the number of manufacturing units was equal to the number of customers. In the second case, the number of manufacturing units was lower than the number of customers. If such a case occurs, it is called a degenerate distribution problem, e.g. three manufacturing units and four customers. With each modification, the calculation correctness is verified by the cost function calculation, where the calculated value for the new solution had to be lower than the previous one. In practice, a degenerate distribution problem is more frequent than the case with an equal number of manufacturing units and customers. In the paper, it is proved that by means of the given algorithms of operational analysis methods it is possible to optimize a current manufacturing process by its replacement for a workshop manufacturing process. The calculations were performed numerically and based on the application of the MS Excel.

\section{COMPLEXES DECOMPOSITION OF THE PROBLEMS}

When providing decomposition,it is necessary to select such sub-complexes. The issues of decomposition are

- The system is expressed by a scheme and tables.

- Tables are decomposed into sub-tables.

- The equations for scheme and sub-tables are defined and calculated.

- The solution connection for sub-tables is provided so that the solution of the original table can be obtainedIn the control system which is called hierarchical calculationand that real corresponds the principal problems are connected with the convergence of the selected algorithm.Convergence of the discontinuous for the method of numerical calculated can be investigated theoretically. When searching the solution of the problem in the off-line than a reduction in the calculations volume.

\section{SYSTEMS CONTROL}

We deal with the following functional factors of control

- Direct control step.

- Optimization step.

- Adaptation step.

- Organization step.

The first step is chracterised by the problem it directly effects the control process. The first control step is very important in the process of data collection at the step of process variables and this information can be different. Information on connected or discrete values which appear, information on the beginning and the end of each phae of a sequential program, information for detection of abnormal situations caused by material defect of a system. The second step is to define more strictlz the problem which is solved by the first step. If the abnormal situation is detected the task of the second step is to activate procedures and to stop the normal function of control (Kanjilal,
P. 1995). The third step is the adaptation step in the broadest sense.The task is to anticipate the transition from the normal mode of operation. The fourth step is the organization step. The decisions are based on at least evaluation of behaviour and global aims of a manufacturing process. All the steps contribute to the effectiveness of a global manufacturing process that is why no one can be omitted. All the steps are interconnected that is why the key issues are communication and coordination.

\section{CONCLUSION}

The problem of the text is the optimization manufacturing process in the real manufacturing workshop. The contribution of this article consists in elaboration of the issue of the processes and complex system control. The solution and its optimization will be testified within further research by other algorithms of operational analysis methods as well (Macura, 2005). At the same time, we will compare a manufacturing process optimization in the given plant. This is the method how the manufacturing program is modified and how selling as well as the profit of the manufacturing plant is provided. Determine of the solutions using the method Kanban by the method operation analysis in the real manufacturing is the new method in the manufacturing process.Some classification aspects when dividing the process in the workshop manufacturing are presented.Methods of decomposition of complexes process as well as the issue of convergence in decomposition are described in the paper.It is shown that the theorz of complex systems control is the new development stage of manufacturing research. The main areas within the issue if the complex systems control manufacturing process are specified.Further, a general procedure transforming the optimiyation manufacturing process under consideration into a form amenable to the iterative algorithms (Hrubina \& Jadlovska, 2002)..The present paper states in a general way the optimal control problem for the manufacturing process as system with distributed parameters whose mathematical model is based on operator equations and inequalities.In general the issue of structure selection consists of two tasks, analysis and synthesis. Because of the complicated nature of the considered control system it is not often possible to describe the operation of a process analyticcally, that is why when describing the function and selecting the structure of control process we often use computer simulation by applying mathematical models.If we consider the authomated process of production control to be the complex system, which consists of the set of hierarchical subprocess operating on space and time, the the two steps of modelling can be distinguished, particular process modelling,whole process.

\section{REFERENCES}

Balczak, S. \& al (2010). Strategy of manufacturing programme optimization in manufacturing system. Annals of DAAAM for 2010 \& Proceedings of the 21th international DAAAM symposium, Vienna, ISBN 978-3-901509-73-5, ISSN 17269679, Katalinic,B. (Ed.), pp. 0563-0564, DAAAM International, Vienna

Hrubina K. \& Jadlovska A. (2002). Optimal Control and Approximation of Variation Inequalities Cybernetics. The International Journal of Systems and Cybernetics. MCB University Press of England. Vol. 31, No 9/10, 2002, 1401 -1408 , ISSN 036-492

Kanjilal, P. (1995). Adaptive prediction and predictive control, IEE Control Engineering, Series 52, pg. 518, Institution Electrical Engineers, ISBN 0863411932, London

Katalinic, B.(1998)Actual Philosophy of Technology in the Search of New Concepts of product and Production. Vienna, ISBN 3-901509-08-9,TU Cluj-Napoca

Macura, D. (2005). Function of Multivariable 1. issue. Presov: FHPV PU, pg. 56, ISBN 80 -8068-321-2 